\title{
A pilot study of chemotherapy and traditional plant medicine in hematology malignancy: Report of thirty-four
} cases

\author{
George Zhu $^{1 *}$, Saboor-Yaraghi $\mathrm{AA}^{2}$, Dharmadhikari $\mathrm{D}^{3}$ and Baer $\mathrm{J}^{4}$ \\ ${ }^{1}$ The Institute of Oncology of George Zhu, china \\ ${ }^{2}$ Department of Celluar and Molecular Nutrition, School of Nutritional Sciences and Dietetics, Tehran University of Medical Sciences, Tehran, Iran \\ ${ }^{3}$ National Blood Transfusion Center, Gaborone, Botswana, South Africa \\ ${ }^{4}$ National Institute of Health, Washington DC-20015, USA
}

\begin{abstract}
Traditional systems of medicine all over the world even traditional medicine and cancer have been using plants and plants products for therapeutic purposes. In this study, 34 patients with hematologic malignancy and other available cancers were entered in the study during 1993-2014.The complete remission (CR) was obtained in 24 of 34 (70.6\%) hematology malignancy and other advanced cancers. During follow up, the survival time in three lung cancer was 10,10 and 17 years, 1 jaundice cholangiocarcinoma was 2 years, 1 gallbladder cancer 15 years, 1 gastric cancer 7 years, 9 lymphoma over $10-20$ years, 1 refractory anemia over 11 years, and 2 chronic granulocytic leukemia (CGL) 5 years and 8 years respectively. CR was once obtained after all-trans retinoic acid(RA) and low dose (1 mg) plant medicine homoharringtonine intravenously in one case of acute promyelocytic leukemia(APL). A CR was achieved by use of chlorambucil and traditional medicine in 1 chronic lymphocytic leukemia with his peripheral leukocytes $123.88 \times 109 /$ L, with a leukocyte differential count of $85 \%$ small lymphocytes. A 62 -year-old female multiple myeloma was in CR after low-dose thalidomide and plant medicine Vinca rosea (Catharanthus roseus). Traditional medicine consisted of Vinca rosea, astragalus membanaceus Bunge, ophiopogon japonicas, asparagus cochinchinensis, angelica sinensis, poria cocos, coix lacryma jobi L. var mayuen, solanum nigrum L, houttuynia cordata, scutellaria barbata d.don and oldenlandia diffusa roxb. During the follow up of three years, she remained CR. In this study, I experienced that a CR was a pivotal influencing factor in those longest survival patients, and traditional medicine was also recommended. As the mechanism of RA to APL treatment, RA (ATRA or cis RA) induce the APL CR and relieve the blockage of promyelocyte differentiation via binding to oncogenic pml/RARa whereas oncogenic pml/ RARa fusion act as a constitutive repressor of RAR and retinoic acid signaling, inducing a differentiation block at promyelocytic stage. This is first described in eukaryotes.
\end{abstract}

\section{Introduction}

Chemotherapy is a major skillful of cancer therapy. One of the most important advances in oncology has been increased acceptance of evidence that most patients with disseminated tumors were settled the protocol of chemotherapy in conjunction with adjuvant traditional plant medicine and target therapy or adoptive immunotherapy (LAK cells).With combination chemotherapy, initial complete remission (CR) was achieved in well over $90 \%$ of acute lymphocytic leukemia in children and in up to $80 \%$ of adults and with survival of 5 years or more seen in over $50 \%$ of children and $10-20 \%$ of adults [1]. At the John Hopkins Oncology Center, with previously untreated de novo acute myelocytic leukemia(AML) in first remission, cures can be achieved in approximately $40 \%$ of patients using either high dose cytarabine regimens or allogenic transplantation BMT following high doses of busulfan and cyclophosphamide(CTX) [2]. The experience in Ugandan children with Hodgkin's disease has been excellent [3] and in a study of 14 adults with stage I and II Hodgkin's disease, mostly clinically staged, 13 patients (93\%) achieved CR with combination chemotherapy and all were in CR 11 to 94 months after completion of treatment [4]. The results will attempt to place in proper interpretative review from those cured patients with hematology malignancy and advanced cancers in my group.

\section{Materials and methods}

34 patients with available cancers were entered in the study during 1993-2014. All patients were in progressive when they were hospitalized. The sex ratio of male: female was 22: 12 respectively. The mean age at onset was 41.5 years (range 11-79 years). The clinical diagnosis of the diseases was lymphoma 13 cases, refractory anemia 1 , acute myelocytic leukemia (FAB:M1 type 2, M2 type 1), acute promyelocytic leukemia (M3) 1, chronic granulocytic leukemia(CGL) 2, chronic lymphocytic leukemia (CLL) 1 , and multiple myeloma 1 case, and other advanced gastric cancer 3 , metastatic lung cancer 6 , thyroid cancer 1 , gallbladder cancer 1 , cholangiocarcinoma 1 case. All patients were treated with the different dosage of combination chemotherapy in conjunction with traditional medicine. The criteria of complete remission (CR) and /or partial remission $(\mathrm{PR})$ is according to the rules where physicians have

Correspondence to: George Zhu, Institute of Oncology of George Zhu, 422407, Beijing, China, E-mail: sansan4240732@163.com

Key words: hematology malignancy, chemotherapy, traditional plant medicine, lymphocytic leukemia

Received: March 02, 2017; Accepted: March 20, 2017; Published: March 22, 2017 
in common with in clinics.

\section{Results}

The complete remission(CR)was obtained in 24 of 34 hematology malignancy and advanced cancers. A $52.9 \%(18 / 34)$ of CR patients was survival over 5 years, 16 patient over 10 years, the longest patients over 20 years. In differential types of 16 patients with 10 years survivors, lymphoma occupied 9 cases (56.2\%).

The objective response of the patients' remission was achieved after one to four courses of treatment. A short CR was achieved by the protocol of FAM or FMC (5-Fu, Ara-c, Mitomycin MC, MTX or CTX) plus cantharidine in 3 advanced gastric cancers. One of them was a long-term survivor for 7 years via mass incision and the combination of FAM with traditional herbs Scutellaria barbata d.don. Remission was indeed efficacy by primary of antitumor compound capsule with traditional herbs plus 5-fluorouracial(5-Fu) injection in a 40 -year-old advanced gastric adenocarcinoma (Figure 1). He was survival over 1 year.

As to approach to 6 lung cancer, a protocol was mainly by usage of traditional medicine and a small dose of chemotherapy. A 64-yearold woman with metastatic lung cancer was in stable in disease for $8+$ months through the combination of compound cantharidine capsule and gefitinib target therapy.

In view of the types of hematology malignancy, lymphoma was settled into the major protocol of the combination conventional chemotherapy. Traditional medicine can relieve the chemotherapeutic toxicity and reinforced the response of chemotherapy. In November 8 , 2013, a 56-year-old patient was entered into clinic due to his lymphoma for $6^{+}$months duration. Physical examination revealed a $3 \times 4.5 \mathrm{~cm}$ lymph node in his left cervical and a $1.5 \times 2 \mathrm{~cm}$ lymph node in right cervical region. The lymph node at his left cervical regressed to $1.5 \times$ $1.3 \mathrm{~cm}$ followed by oral compound cantharidine and another drug Pingxiao tablets. At the follow up of 3 years, he was in stable disease now.

There were 3 acute leukemias (M1 2 cases, M3 1 case) with a fatal infection and fever at onset of the serious disease, and a higher fever falls to normal when patients receiving both forms of traditional medicine and antibiotics regimen in full dose and small dosage of dexamethasone. In one 18-year-old girl, remission was obtained by DNR (daunorubicin) $40 \mathrm{mg} / \mathrm{m}^{2}$ (days 1 to 3) and Ara-c(A) $200 \mathrm{mg} / \mathrm{m}^{2}$ (days 1 to 4), followed by one month of traditional medicine. She had a $92 \%$ blasts in a $\sim 90 \%$ cellular marrow and a $91 \%$ blasts in peripheral differentiate leukocytes at onset of newly diagnosed acute leukemia, and $15.1 \%$ blasts plus promyelocytes in normal cellular marrow and $2 \%$ blasts plus promyelocytes after DA chemotherapy. Another a

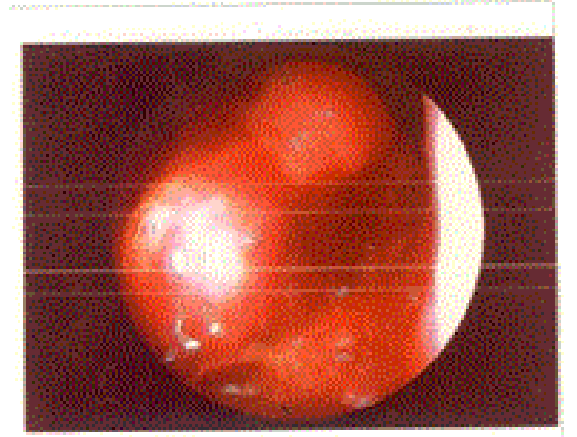

Figure 1. At gastroscope show a $1.2 \times 0.8 \mathrm{~cm}$ nodular ulcer in a 40 -year old patient with adenocarcinoma. 20-year-old M2 patient, PR was obtained by homoharringtonine (H)4 $\mathrm{mg} / \mathrm{m}^{2}$ (days 1 to 3 ) and Ara-c $50 \mathrm{mg}$ intramuscle injection, twice a day(days 1 to 5). After two courses of HA combination chemotherapy, a $71.26 \%$ blasts plus promyelocytes in a $\sim 90 \%$ cellular marrow at onset of acute leukemia was declined to $33 \%$ (71.26\% vs 33\%) blasts plus promyelocytes in a $\sim 70 \%$ cellular marrow whereas no immature cells were found in peripheral differentiate leukocytes compared to a $72.1 \%(72.1 \%$ vs $0 \%)$ immature cells at onset of disease. A 62-year-old woman with multiple myeloma was in CR after low-dose thalidomide and plant medicine. She was an over 3 years survivor.

\section{Case reports}

A 20-year-old man who entered the hospital owing to his tooth bruise and generalized lymphadenopathy on April 19,1996. He had once the history of axillary lymphadectomy in other hospital due to axillary lymphadenopathy and marked splenic enlargement in 1994.

At physical examination, the patient was tooth bruise and its bleeding. Both sides of the cervical $(6 \times 5 \mathrm{~cm}, 2 \times 3 \mathrm{~cm})$ and right inguinal $(4.5 \times 2.5 \mathrm{~cm})$ lymph nodes were palpable and shorty. The marked splenomegaly beneath the umbilicus was easily palpable during examination. On " $\mathrm{B}$ ” ultrasound examination was the presentation of splenic-type lymphoma and splenomegaly.

Hemoglobin concentration was $10 \mathrm{~g} / \mathrm{L}$.Leukocyte count was $7.4 \times$ 109/L. The platelet count was $60 \times 109 /$ L. Bone marrow examination of aspirates revealed normal cellularity with abnormal histocytosis $(\sim 20 \%)$. The diagnosis of chronic histocytosis and splenic-type lymphoma was made.

$\mathrm{CR}$ was achieved by the use of the conventional chemotherapy (VCR, CTX,5-Fu, MC) in conjunction with traditional medicine. Four months later the lymphadenopathy recurred again, accompanied with the symptoms of fever and two lymph nodes (thumb size) in right cervical region. The patient received the continuous chemotherapy. During treatment with COM (VCR, CTX, MC) plan, the lymphadenopathy regressed, but a $1.5 \times 1.5 \mathrm{~cm}$ lymph node can't completely be in regression. Oral 5-Fu was administered in a total dose of 5 gram (100\#), with complete regression of his lymphadenopathy. $\mathrm{He}$ was in disease-free survivor for 13 years, and had health child. In April, 2010, he died of primary hepatocellular carcinoma(PHC).

A 34-year-old man was admitted into the hospital due to his left submaxillary lymphadenopathy for 3 months duration on March 2, 1997. At physical examination showed $2 \mathrm{~cm} \times 3 \mathrm{~cm}$ and $3 \mathrm{~cm} \times 3.5 \mathrm{~cm}$, and $3 \mathrm{~cm} \times 4 \mathrm{~cm}$ lymph nodes palpable and shorty in his left cervical region. NHL was diagnosed according to his lymph nodes aspirates. Cures was achieved after 3 courses of combination conventional chemotherapy (VCR, CTX, adriamycin ADM and dexamethasone Dex) in conjunction with traditional medicine. And in 13 years later he remained well.

A 31-year-old man who entered hospital on October 16, 2003 because of fever, irritability and pallor of 15 days duration. Physical examination revealed marked pallor, hepatosplenomegaly. Persistent fever reached to $39.5 \mathrm{C}$. Chest X-ray showed small amount of hydrothorax. Liver CT scan demonstrated an elliptical mass of $7.0 \mathrm{~cm}$ $\times 4.5 \mathrm{~cm}$ which was considered to secondary hepatic malignant tumor. AFP negative.

Hemoglobin concentration was 53g/L.leukocyte count $3.4 \times 109 / \mathrm{L}$ with $20 \%$ of promyelocytes. The platelet count was $2.4 \times 109 /$ L.Bone marrow aspiration revealed normal cellularity. Approximately $77 \%$ of 
marrow cells were promyelocytes. The diagnosis of acute promyelocytic leukemia(APL) complicated with hepatoma was made.

Treatment consisted of $80 \mathrm{mg}$ per day of retinoic acid (RA) and $1 \mathrm{mg}$ of homoharringtonine intravenously per day for 5 days. High fever was declined to normal after receiving traditional medicine and small dose of dexamethasone. CR was obtained after all-trans RA in conjunction with traditional medicine and chemotherapy one month duration treatment. The liver scan showed the disappearance of hepatic tumor.He was still in CR 1 year later.

\section{Discussion}

In this study, a series of the long follow up of those cured patients with hematology malignancy and other advanced cancers were reported. I experienced that a CR was a pivotal influencing factor in those longest survival patients, and traditional plant medicine was also recommended.

As a novel RA to APL treatment, RA (ATRA or cis RA) induce APL $\mathrm{CR}$ and relieve the blockage of promyelocyte differentiation via binding to oncogenic pml/RARa whereas oncogenic pml/RARa fusion act as a constitutive repressor of RAR and retinoic acid signaling, inducing a differentiation block at promyelocytic stage [5-8]. This is first described in eukaryotes.

\section{References}

1. Henderson ES (1983) Acute lymphocytic leukemia In:Gunz FW, Henderson ES(Eds) Leukemia in fourth edition pp. 575-625.

2. Geller RB, Saral R, Karp JE, Santos GW, Burke PJ (1990) Cure of acute myelocytic leukemia in adults: a reality. Leukemia 4: 313-315.[Crossref]

3. Olweny CL, Katongole-Mbidde E, Kiire C, Lwanga SK, Magrath I, et al. (1978) Childhood Hodgkin's disease in Uganda: a ten-year experience. Cancer 42: 787-792. [Crossref]

4. Lauria F, Baccarani M, Fiaccini M, Mazza P, Tura S (1979) Combination chemotherapy in stages I or II Hodgkin's disease. Lancet 2: 1072-1073.[Crossref]

5. Zhu G (2013) Novel treatment of acute promyelocytic leukemia: $\mathrm{As}_{2} \mathrm{O}_{3}$, retinoic acid and retinoid pharmacology. Curr Pharm Biotechnol 14: 849-858.[Crossref]

6. Zhu G,Saboor-Yaraghi AA,Yarden Y, Santos J, Neil JC (2016) Downregulating oncogenic receptor:From bench to clinic. Hematol Med Oncol1:30-40.

7. Zhu G,Saboor-Yaraghi AA,Yarden Y (2017) Targeting oncogenic receptor:From molecular physiology to currently the standard of target therapy. Advances Pharmaceutical Journal 2: 10-28.

8. Zhu G,Saboor-Yaraghi AA,Yarden Y, Baer J (2017) Targeting oncogenic receptor,currently the standard of care. American Journal of Clinical and Experimental Medicine 6:(in press).

Copyright: (C2017 Zhu G. This is an open-access article distributed under the terms of the Creative Commons Attribution License, which permits unrestricted use, distribution, and reproduction in any medium, provided the original author and source are credited. 